\title{
Conduction-Convection-Radiation Heat Transfer in High Temperature Catalytic Reactors
}

\author{
Georg Liesche ${ }^{1}$, Kai Sundmacher ${ }^{1,2}$ \\ ${ }^{1}$ Max-Planck-Institute for Dynamics of Complex Technical Systems \\ Sandtorstr. 1, 39106 Magdeburg, Germany \\ liesche@mpi-magdeburg.mpg.de; sundmacher@mpi-magdeburg.mpg.de \\ ${ }^{2}$ Otto-von-Guericke University \\ Universitätsplatz 2, 39106 Magdeburg, Germany
}

\section{Extended Abstract}

In this contribution, the analysis of heat transfer in a high temperature catalytic gas phase reactor is carried out based on a first-principles modelling approach combined with engineering heuristics using dimensionless numbers. Typical examples include steam methane reforming (SMR) and synthesis of hydrogen cyanide where highly endothermic reactions $\left(\Delta_{R} \mathrm{~h}>250 \mathrm{~kJ} / \mathrm{mol}\right)$ take place on catalytic surfaces of tubes or channels that are placed in a furnace to provide the high reaction enthalpy. Key design aspect for such reactor systems is the heat transfer from the hot flue gases to the active catalytic sites of the synthesis compartments. Understanding the significance of each of the three modes of heat transfer paves the way towards reactor optimisation and process intensification reducing the resource footprint of the industry.

The strong coupling of convection, conduction and radiation is investigated with a rigorous two-dimensional fluiddynamical model consisting of partial differential equations (PDEs) for total continuity, partial continuity, momentum, energy, and radiative intensity via the radiative transfer equation (RTE). The equations are discretized in finite volumes and the resulting differential algebraic system is solved in Matlab/CasADi to maintain full access to the formulation of the equations. The underlying physical modelling assumptions of the reactor model are scrutinized carefully: The relevant physical transport phenomena are identified with a combination of a dimensionless analysis using Reynolds, Grashoff, Boltzmann and Planck numbers and their numerical equivalents from in silica studies with the reactor model with special emphasis on heat transfer which is crucial for high temperature applications [1]. Radiative heat transfer of the multicomponent gas mixture is accounted for using the Planck mean absorption coefficient and thus a quantitative comparison of all three modes of heat transfer is achieved for this reactive gas flow. The lack of absorptive data for the reacting species - in particular ammonia and $\mathrm{HCN}$ - is overcome via the application of the spectroscopic absorption database HITRAN to obtain temperature and species-dependent absorption coefficients [2]. The importance of both substance and temperature dependence is illustrated - neglecting any dependence and assuming constant values leads to strong under- or overestimation of the importance of radiation. Dimensionless numbers are used as indicators for identification of relevant transport phenomena in the reactor. Their estimates are compared with modelling results and it is illustrated why the dimensionless approach is not necessarily successful particularly when radiation is taken into account. The resulting model is validated against industrial and literature data for the case study of hydrogen cyanide (HCN) synthesis from methane and ammonia providing an excellent agreement among all data. Flow regimes that are dominated by one of the three heat transfer modes are identified and highlighted in the presentation providing the initial step for further process intensification and high temperature gas phase reactor design optimisation opportunities [3].

\section{References}

[1] E. Koberstein, "Model reactor studies of the hydrogen cyanide synthesis from methane and ammonia," Ind. Eng. Chem. Process Des. Develop., vol. 12, no. 423 pp. 444-448, 1973.

[2] R. V. Kochanov, I. E. Gordon, L. S. Rothman, P. Wcis lo, C. Hill, J. S. Wilzewski, "Hitran application programming interface (hapi): A comprehensive approach to working with spectroscopic data," J. of Quant. Spec. and Rad. Tr., vol. 177, pp. 15-30, 2016. 
[3] G. Liesche, K. Sundmacher, "Scrutinising Optimal Design of High Temperature Reactors: Flow and Heat Transfer Characteristics," I\&ECR, submitted. 